\title{
A REFINEMENT OF THE GRÜSS INEQUALITY AND APPLICATIONS
}

\author{
P. CERONE AND S. S. DRAGOMIR
}

\begin{abstract}
A sharp refinement of the Grüss inequality in the general setting of measurable spaces and abstract Lebesgue integrals is proven. Some consequential particular inequalities are mentioned.
\end{abstract}

\section{Introduction}

Let $(\Omega, \mathcal{A}, \mu)$ be a measurable space consisting of a set $\Omega$, a $\sigma$ - algebra $\mathcal{A}$ of parts of $\Omega$ and a countably additive and positive measure $\mu$ on $\mathcal{A}$ with values in $\mathbb{R} \cup\{\infty\}$.

For a $\mu$-measurable function $w: \Omega \rightarrow \mathbb{R}$, with $w(x) \geq 0$ for $\mu$-a.e. $x \in \Omega$, consider the Lebesgue space $L_{w}(\Omega, \mu):=\left\{f: \Omega \rightarrow \mathbb{R}, f\right.$ is $\mu$-measurable and $\int_{\Omega} w(x)|f(x)| d \mu(x)<$ $\infty\}$. Assume $\int_{\Omega} w(x) d \mu(x)>0$.

If $f, g: \Omega \rightarrow \mathbb{R}$ are $\mu$-measurable functions and $f, g, f g \in L_{w}(\Omega, \mu)$, then we may consider the Čebyšev functional

$$
\begin{aligned}
T_{w}(f, g):= & \frac{1}{\int_{\Omega} w(x) d \mu(x)} \int_{\Omega} w(x) f(x) g(x) d \mu(x) \\
& -\frac{1}{\int_{\Omega} w(x) d \mu(x)} \int_{\Omega} w(x) f(x) d \mu(x) \\
& \times \frac{1}{\int_{\Omega} w(x) d \mu(x)} \int_{\Omega} w(x) g(x) d \mu(x) .
\end{aligned}
$$

The following result is known in the literature as the Grüss inequality

$$
\left|T_{w}(f, g)\right| \leq \frac{1}{4}(\Gamma-\gamma)(\Delta-\delta),
$$

provided

$$
-\infty<\gamma \leq f(x) \leq \Gamma<\infty, \quad-\infty<\delta \leq g(x) \leq \Delta<\infty
$$

for $\mu$ - a.e. $x \in \Omega$.

Received May 31, 2005.

2000 Mathematics Subject Classification. Primary 26D15, 26D20; Secondary 26D10.

Key words and phrases. Grüss inequality, measurable functions, Lebesgue integral, perturbed rules. 
The constant $\frac{1}{4}$ is sharp in the sense that it cannot be replaced by a smaller constant.

Note that if $\Omega=\{1, \ldots, n\}$ and $\mu$ is the discrete measure on $\Omega$, then we obtain the discrete Grüss inequality

$$
\left|\frac{1}{W_{n}} \sum_{i=1}^{n} w_{i} x_{i} y_{i}-\frac{1}{W_{n}} \sum_{i=1}^{n} w_{i} x_{i} \cdot \frac{1}{W_{n}} \sum_{i=1}^{n} w_{i} y_{i}\right| \leq \frac{1}{4}(\Gamma-\gamma)(\Delta-\delta),
$$

provided $\gamma \leq x_{i} \leq \Gamma, \delta \leq y_{i} \leq \Delta$ for each $i \in\{1, \ldots, n\}$ and $w_{i} \geq 0$ with $W_{n}:=$ $\sum_{i=1}^{n} w_{i}>0$.

The following result was proved in Cheng and Sun [4].

Theorem 1. Let $f, g:[a, b] \rightarrow \mathbb{R}$ be two integrable functions such that $\delta \leq g(x) \leq \Delta$ for some constants $\delta, \Delta$ for all $x \in[a, b]$, then

$$
\begin{gathered}
\left|\frac{1}{b-a} \int_{a}^{b} f(x) g(x) d x-\frac{1}{(b-a)^{2}} \int_{a}^{b} f(x) d x \int_{a}^{b} g(x) d x\right| \\
\leq \frac{\Delta-\delta}{2} \frac{1}{b-a} \int_{a}^{b}\left|f(x)-\frac{1}{b-a} \int_{a}^{b} f(y) d y\right| d x .
\end{gathered}
$$

They used the result (1.5) to obtain perturbed trapezoidal rules.

In the current paper we obtain bounds for $\left|T_{w}(f, g)\right|$ under the general setting expressed in (1.1). A bound which is shown to be sharp is obtained in Section 2. The sharpness of (1.5) was not demonstrated in [4]. Sharp results were obtained for a perturbed interior point rule (Ostrowski-Grüss) inequalities in Cheng [3]. Some particular instances of the results in Section 2 are investigated in Sections 4 and 5, recapturing earlier work. Results are presented in Section 3, for Lebesgue measurable functions and for a discrete weighted Čebyšev functional involving $n$-tuples.

\section{An integral inequality}

With the assumptions as presented in the Introduction and if $f \in L_{w}(\Omega, \mu)$ then we may define

$$
\begin{aligned}
D_{w}(f):= & D_{w, 1}(f) \\
:= & \frac{1}{\int_{\Omega} w(x) d \mu(x)} \int_{\Omega} w(x) \\
& \times\left|f(x)-\frac{1}{\int_{\Omega} w(y) d \mu(y)} \int_{\Omega} w(y) f(y) d \mu(y)\right| d \mu(x) .
\end{aligned}
$$

The following fundamental result holds.

Theorem 2. Let $w, f, g: \Omega \rightarrow \mathbb{R}$ be $\mu$-measurable functions with $w \geq 0 \mu$-a.e. on $\Omega$ and $\int_{\Omega} w(y) d \mu(y)>0$. If $f, g, f g \in L_{w}(\Omega, \mu)$ and there exists the constants $\delta, \Delta$ such that

$$
-\infty<\delta \leq g(x) \leq \Delta<\infty \text { for } \mu-\text { a.e. } x \in \Omega
$$


then we have the inequality

$$
\left|T_{w}(f, g)\right| \leq \frac{1}{2}(\Delta-\delta) D_{w}(f)
$$

The constant $\frac{1}{2}$ is sharp in the sense that it cannot be replaced by a smaller quantity.

Proof. Obviously, we have

$$
\begin{aligned}
T_{w}(f, g)= & \frac{1}{\int_{\Omega} w(x) d \mu(x)} \int_{\Omega} w(x) \\
& \times\left(f(x)-\frac{1}{\int_{\Omega} w(y) d \mu(y)} \int_{\Omega} f(y) w(y) d \mu(y)\right) g(x) d \mu(x) .
\end{aligned}
$$

Consider the measurable subsets $\Omega_{+}$and $\Omega_{-}$, of $\Omega$, defined by

$$
\Omega_{+}:=\left\{x \in \Omega \mid f(x)-\frac{1}{\int_{\Omega} w(y) d \mu(y)} \int_{\Omega} w(y) f(y) d \mu(y) \geq 0\right\}
$$

and

$$
\Omega_{-}:=\left\{x \in \Omega \mid f(x)-\frac{1}{\int_{\Omega} w(y) d \mu(y)} \int_{\Omega} w(y) f(y) d \mu(y)<0\right\} .
$$

Obviously, $\Omega=\Omega_{+} \cup \Omega_{-}, \Omega_{+} \cap \Omega_{-}=\emptyset$ and if we define

$$
\begin{aligned}
I_{+}(f, g, w):= & \frac{1}{\int_{\Omega} w(y) d \mu(y)} \int_{\Omega_{+}} w(x) \\
& \times\left(f(x)-\frac{1}{\int_{\Omega} w(y) d \mu(y)} \int_{\Omega} w(y) f(y) d \mu(y)\right) g(x) d \mu(x)
\end{aligned}
$$

and

$$
\begin{aligned}
I_{-}(f, g, w):= & \frac{1}{\int_{\Omega} w(y) d \mu(y)} \int_{\Omega_{-}} w(x) \\
& \times\left(f(x)-\frac{1}{\int_{\Omega} w(y) d \mu(y)} \int_{\Omega} w(y) f(y) d \mu(y)\right) g(x) d \mu(x)
\end{aligned}
$$

then we have

$$
T_{w}(f, g)=I_{+}(f, g, w)+I_{-}(f, g, w) .
$$

Since $-\infty<\delta \leq g(x) \leq \Delta<\infty$ for $\mu$ - a.e. $x \in \Omega$ and $w(x) \geq 0$ for $\mu$-a.e. $x \in \Omega$, we may write:

$$
\begin{aligned}
I_{+}(f, g, w) \leq & \frac{\Delta}{\int_{\Omega} w(y) d \mu(y)} \int_{\Omega_{+}} w(x) \\
& \times\left(f(x)-\frac{1}{\int_{\Omega} w(y) d \mu(y)} \int_{\Omega} f(y) w(y) d \mu(y)\right) d \mu(x)
\end{aligned}
$$


and

$$
\begin{aligned}
I_{-}(f, g, w) \leq & \frac{\delta}{\int_{\Omega} w(y) d \mu(y)} \int_{\Omega_{-}} w(x) \\
& \times\left(f(x)-\frac{1}{\int_{\Omega} w(y) d \mu(y)} \int_{\Omega} f(y) w(y) d \mu(y)\right) d \mu(x) .
\end{aligned}
$$

Since

$$
\begin{aligned}
0= & \int_{\Omega} w(x)\left(f(x)-\frac{1}{\int_{\Omega} w(y) d \mu(y)} \int_{\Omega} f(y) w(y) d \mu(y)\right) d \mu(x) \\
= & \int_{\Omega_{+}} w(x)\left(f(x)-\frac{1}{\int_{\Omega} w(y) d \mu(y)} \int_{\Omega} f(y) w(y) d \mu(y)\right) d \mu(x) \\
& +\int_{\Omega_{-}} w(x)\left(f(x)-\frac{1}{\int_{\Omega} w(y) d \mu(y)} \int_{\Omega} f(y) w(y) d \mu(y)\right) d \mu(x)
\end{aligned}
$$

we get

$$
\begin{aligned}
\int_{\Omega_{-}} & w(x)\left(f(x)-\frac{1}{\int_{\Omega} w(y) d \mu(y)} \int_{\Omega} f(y) w(y) d \mu(y)\right) d \mu(x) \\
= & -\int_{\Omega_{+}} w(x)\left(f(x)-\frac{1}{\int_{\Omega} w(y) d \mu(y)} \int_{\Omega} f(y) w(y) d \mu(y)\right) d \mu(x)
\end{aligned}
$$

and thus, from (2.7), we deduce

$$
\begin{aligned}
I_{-}(f, g, w) \leq & \frac{-\delta}{\int_{\Omega} w(y) d \mu(y)} \int_{\Omega_{+}} w(x) \\
& \times\left(f(x)-\frac{1}{\int_{\Omega} w(y) d \mu(y)} \int_{\Omega} w(y) f(y) d \mu(y)\right) d \mu(x) .
\end{aligned}
$$

Consequently, by adding (2.6) with (2.8), we deduce

$$
\begin{aligned}
T_{w}(f, g) \leq & \frac{\Delta-\delta}{\int_{\Omega} w(y) d \mu(y)} \int_{\Omega_{+}} w(x) \\
& \times\left(f(x)-\frac{1}{\int_{\Omega} w(y) d \mu(y)} \int_{\Omega} w(y) f(y) d \mu(y)\right) d \mu(x) .
\end{aligned}
$$

On the other hand,

$$
\begin{aligned}
\int_{\Omega} w(x)\left|f(x)-\frac{1}{\int_{\Omega} w(y) d \mu(y)} \int_{\Omega} f(y) w(y) d \mu(y)\right| d \mu(x) \\
=\int_{\Omega_{+}} w(x)\left|f(x)-\frac{1}{\int_{\Omega} w(y) d \mu(y)} \int_{\Omega} f(y) w(y) d \mu(y)\right| d \mu(x) \\
\quad+\int_{\Omega_{-}} w(x)\left|f(x)-\frac{1}{\int_{\Omega} w(y) d \mu(y)} \int_{\Omega} f(y) w(y) d \mu(y)\right| d \mu(x)
\end{aligned}
$$




$$
\begin{aligned}
= & \int_{\Omega_{+}} w(x)\left(f(x)-\frac{1}{\int_{\Omega} w(y) d \mu(y)} \int_{\Omega} f(y) w(y) d \mu(y)\right) d \mu(x) \\
& -\int_{\Omega_{-}} w(x)\left(f(x)-\frac{1}{\int_{\Omega} w(y) d \mu(y)} \int_{\Omega} f(y) w(y) d \mu(y)\right) d \mu(x) \\
= & 2 \int_{\Omega_{+}} w(x)\left(f(x)-\frac{1}{\int_{\Omega} w(y) d \mu(y)} \int_{\Omega} f(y) w(y) d \mu(y)\right) d \mu(x),
\end{aligned}
$$

and thus, by (2.9) we deduce

$$
\begin{aligned}
T_{w}(f, g) \leq & \frac{1}{2}(\Delta-\delta) \frac{1}{\int_{\Omega} w(y) d \mu(y)} \int_{\Omega} w(x) \\
& \times\left|f(x)-\frac{1}{\int_{\Omega} w(y) d \mu(y)} \int_{\Omega} f(y) w(y) d \mu(y)\right| d \mu(x) .
\end{aligned}
$$

Now, if we write the inequality (2.10) for $-f$ instead of $f$ and taking into account that $T_{w}(-f, g)=-T_{w}(f, g)$, we deduce

$$
\begin{aligned}
-T(f, g) \leq & \frac{1}{2}(\Delta-\delta) \frac{1}{\int_{\Omega} w(y) d \mu(y)} \int_{\Omega} w(x) \\
& \times\left|f(x)-\frac{1}{\int_{\Omega} w(y) d \mu(y)} \int_{\Omega} f(y) w(y) d \mu(y)\right| d \mu(x),
\end{aligned}
$$

giving the desired inequality (2.3).

To prove the sharpness of the constant $\frac{1}{2}$, assume that (2.3) holds for $\Omega=[a, b]$ and $w \equiv 1$, with a constant $C>0$. That is,

$$
|T(f, g)| \leq C(\Delta-\delta) \frac{1}{b-a} \int_{a}^{b}\left|f(x)-\frac{1}{b-a} \int_{a}^{b} f(y) d y\right| d x
$$

where

$$
T(f, g)=\frac{1}{b-a} \int_{a}^{b} f(x) g(x) d x-\frac{1}{b-a} \int_{a}^{b} f(x) d x \cdot \frac{1}{b-a} \int_{a}^{b} g(x) d x
$$

and the integral $\int_{a}^{b}$ is the usual Lebesgue integral on $[a, b]$.

Choose in (2.12) $g=f$ and $f:[a, b] \rightarrow \mathbb{R}$ defined by

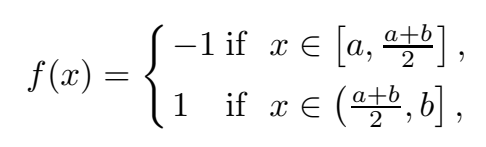

then, obviously,

$$
T(f, f)=\frac{1}{b-a} \int_{a}^{b} f^{2}(x) d x-\left(\frac{1}{b-a} \int_{a}^{b} f(x) d x\right)^{2}=1,
$$




$$
\begin{aligned}
D(f) & =\frac{1}{b-a} \int_{a}^{b}\left|f(x)-\frac{1}{b-a} \int_{a}^{b} f(y) d y\right| d x=1, \\
\delta & =-1, \quad \Delta=1
\end{aligned}
$$

and by (2.12) we get $2 C \geq 1$ giving $C \geq \frac{1}{2}$.

For $f \in L_{p, w}(\Omega, \mathcal{A}, \mu):=\left\{f: \Omega \rightarrow \mathbb{R}, \int_{\Omega} w(x)|f(x)|^{p} d \mu(x)<\infty\right\}, p \geq 1$ we may also define

$$
\begin{aligned}
D_{w, p}(f):= & {\left[\frac{1}{\int_{\Omega} w(x) d \mu(x)} \int_{\Omega} w(x)\right.} \\
& \left.\times\left|f(x)-\frac{1}{\int_{\Omega} w(y) d \mu(y)} \int_{\Omega} w(y) f(y) d \mu(y)\right|^{p} d \mu(x)\right]^{\frac{1}{p}} \\
= & \frac{\left\|f-\frac{1}{\int_{\Omega} w d \mu} \int_{\Omega} w f d \mu\right\|_{\Omega, p}}{\left[\int_{\Omega} w(x) d \mu(x)\right]^{\frac{1}{p}}}
\end{aligned}
$$

where $\|\cdot\|_{\Omega, p}$ is the usual $p$-norm on $L_{p, w}(\Omega, \mathcal{A}, \mu)$, namely,

$$
\|h\|_{\Omega, p}:=\left(\int_{\Omega} w|h|^{p} d \mu\right)^{\frac{1}{p}}, \quad p \geq 1 .
$$

Using Hölder's inequality we get

$$
D_{w, 1}(f) \leq D_{w, p}(f) \text { for } p \geq 1, f \in L_{p, w}(\Omega, \mathcal{A}, \mu) ;
$$

and, in particular for $p=2$

$$
D_{w, 1}(f) \leq D_{w, 2}(f)=\left[\frac{\int_{\Omega} w f^{2} d \mu}{\int_{\Omega} w d \mu}-\left(\frac{\int_{\Omega} w f d \mu}{\int_{\Omega} w d \mu}\right)^{2}\right]^{\frac{1}{2}},
$$

if $f \in L_{2, w}(\Omega, \mathcal{A}, \mu)$.

For $f \in L_{\infty}(\Omega, \mathcal{A}, \mu):=\left\{f: \Omega \rightarrow \mathbb{R},\|f\|_{\Omega, \infty}:=\right.$ ess $\left.\sup _{x \in \Omega}|f(x)|<\infty\right\}$ we also have

$$
D_{w, p}(f) \leq D_{w, \infty}(f):=\left\|f-\frac{1}{\int_{\Omega} w d \mu} \int_{\Omega} w f d \mu\right\|_{\Omega, \infty} .
$$

The following corollary may be useful in practice.

Corollary 1. With the assumptions of Theorem 2, we have

$$
\begin{aligned}
\left|T_{w}(f, g)\right| & \leq \frac{1}{2}(\Delta-\delta) D_{w}(f) \\
& \leq \frac{1}{2}(\Delta-\delta) D_{w, p}(f) \text { if } f \in L_{p}(\Omega, \mathcal{A}, \mu), 1<p<\infty
\end{aligned}
$$




$$
\leq \frac{1}{2}(\Delta-\delta)\left\|f-\frac{1}{\int_{\Omega} w d \mu} \int_{\Omega} w f d \mu\right\|_{\Omega, \infty} \quad \text { if } f \in L_{\infty}(\Omega, \mathcal{A}, \mu) .
$$

Remark 1. The inequalities in (2.17) are in order of increasing coarseness. If we assume that $-\infty<\gamma \leq f(x) \leq \Gamma<\infty$ for $\mu$ - a.e. $x \in \Omega$, then by the Grüss inequality for $g=f$ we have for $p=2$

$$
\left[\frac{\int_{\Omega} w f^{2} d \mu}{\int_{\Omega} w d \mu}-\left(\frac{\int_{\Omega} w f d \mu}{\int_{\Omega} w d \mu}\right)^{2}\right]^{\frac{1}{2}} \leq \frac{1}{2}(\Gamma-\gamma) .
$$

By (2.17), we deduce the following sequence of inequalities

$$
\begin{aligned}
\left|T_{w}(f, g)\right| & \leq \frac{1}{2}(\Delta-\delta) \frac{1}{\int_{\Omega} w d \mu} \int_{\Omega} w\left|f-\frac{1}{\int_{\Omega} w d \mu} \int_{\Omega} w f d \mu\right| d \mu \\
& \leq \frac{1}{2}(\Delta-\delta)\left[\frac{\int_{\Omega} w f^{2} d \mu}{\int_{\Omega} w d \mu}-\left(\frac{\int_{\Omega} w f d \mu}{\int_{\Omega} w d \mu}\right)^{2}\right]^{\frac{1}{2}} \\
& \leq \frac{1}{4}(\Delta-\delta)(\Gamma-\gamma)
\end{aligned}
$$

for $f, g: \Omega \rightarrow \mathbb{R}, \mu$ - measurable functions and so that $-\infty<\gamma \leq f(x)<\Gamma<\infty$, $-\infty<\delta \leq g(x) \leq \Delta<\infty$ for $\mu$ - a.e. $x \in \Omega$. Thus, the inequality (2.19) is a refinement of Grüss' inequality (1.2).

It is well known that if $f \in L_{2, w}(\Omega, \mathcal{A}, \mu)$, then the following Schwarz's type inequality holds:

$$
\frac{1}{\int_{\Omega} w d \mu} \int_{\Omega} w f^{2} d \mu \geq\left(\frac{1}{\int_{\Omega} w d \mu} \int_{\Omega} w f d \mu\right)^{2} .
$$

Using the above results, we may point out the following counterpart result.

Proposition 1. Assume that the $\mu$-measurable function $f: \Omega \rightarrow \mathbb{R}$ satisfies the assumption:

$$
-\infty<\gamma \leq f(x) \leq \Gamma<\infty \text { for a.e. } x \in \Omega .
$$

Then one has the inequality

$$
\begin{aligned}
0 & \leq \frac{1}{\int_{\Omega} w d \mu} \int_{\Omega} w f^{2} d \mu-\left(\frac{1}{\int_{\Omega} w d \mu} \int_{\Omega} w f d \mu\right)^{2} \\
& \leq \frac{1}{2}(\Gamma-\gamma) \frac{1}{\int_{\Omega} w d \mu} \int_{\Omega} w\left|f-\frac{1}{\int_{\Omega} w d \mu} \int_{\Omega} w f d \mu\right| d \mu \\
& \left(\leq \frac{1}{4}(\Gamma-\gamma)^{2}\right) .
\end{aligned}
$$

The constant $\frac{1}{2}$ is sharp. 
The proof follows by the inequality (2.3) for $g=f$.

The following proposition also holds.

Proposition 2. Assume that the measurable functions $f, g: \Omega \rightarrow \mathbb{R}$ satisfy (1.3) (the condition in Grüss' inequality). Then

$$
\begin{aligned}
\left|T_{w}(f, g)\right| & \leq \frac{1}{2}[(\Gamma-\gamma)(\Delta-\delta)]^{\frac{1}{2}}\left[D_{w}(f) D_{w}(g)\right]^{\frac{1}{2}} \\
& \leq \frac{1}{4}(\Delta-\delta)(\Gamma-\gamma) .
\end{aligned}
$$

The constant $\frac{1}{2}$ in the first inequality and $\frac{1}{4}$ in the second inequality are sharp.

Proof. By (2.19) we have

$$
\left|T_{w}(f, g)\right| \leq \frac{1}{2}(\Delta-\delta) D_{w}(f)
$$

and

$$
\left|T_{w}(f, g)\right| \leq \frac{1}{2}(\Gamma-\gamma) D_{w}(g)
$$

from which, by multiplication, gives the first part of (2.23).

The second part and the sharpness of the constants are obvious.

\section{Some particular inequalities}

The following particular inequalities are of interest.

1. Let $w, f, g:[a, b] \rightarrow \mathbb{R}$ be Lebesgue measurable functions with $w \geq 0$ a.e. on $[a, b]$ and $\int_{a}^{b} w(y) d y>0$. If $f, g, f g \in L_{w}[a, b]$, where

$$
L_{w}[a, b]:=\left\{f:[a, b] \rightarrow \mathbb{R}\left|\int_{a}^{b} w(x)\right| f(x) \mid d x<\infty\right\}
$$

and

$$
-\infty<\delta \leq g(x) \leq \Delta<\infty \text { for a.e. } x \in[a, b],
$$

then we have the inequalities

$$
\begin{aligned}
& \mid \frac{1}{\int_{a}^{b} w(x) d x} \int_{a}^{b} w(x) f(x) g(x) d x \\
& \quad-\frac{1}{\int_{a}^{b} w(x) d x} \int_{a}^{b} w(x) f(x) d x \cdot \frac{1}{\int_{a}^{b} w(x) d x} \int_{a}^{b} w(x) g(x) d x \mid \\
& \quad \leq \frac{1}{2}(\Delta-\delta) \frac{1}{\int_{a}^{b} w(x) d x} \int_{a}^{b} w(x)\left|f(x)-\frac{1}{\int_{a}^{b} w(y) d y} \int_{a}^{b} w(y) f(y) d y\right| d x
\end{aligned}
$$




$$
\begin{aligned}
& \leq \frac{1}{2}(\Delta-\delta)\left[\frac{\int_{a}^{b} w(x)\left|f(x)-\frac{1}{\int_{a}^{b} w(y) d y} \int_{a}^{b} w(y) f(y) d y\right|^{p} d x}{\int_{a}^{b} w(x) d x}\right]^{\frac{1}{p}} \\
& \quad \text { if } f \in L_{p, w}[a, b], \quad 1<p<\infty \\
& \leq \frac{1}{2}(\Delta-\delta) \text { ess } \sup _{x \in[a, b]}\left|f(x)-\frac{1}{\int_{a}^{b} w(y) d y} \int_{a}^{b} w(y) f(y) d y\right| \text { if } f \in L_{\infty}[a, b] .
\end{aligned}
$$

The constant $\frac{1}{2}$ is sharp in the first inequality in (3.2).

The following counterpart of Schwarz's inequality holds

$$
\begin{aligned}
0 & \leq \frac{1}{\int_{a}^{b} w(y) d y} \int_{a}^{b} w(x) f^{2}(x) d x-\left(\frac{1}{\int_{a}^{b} w(y) d y} \int_{a}^{b} w(x) f(x) d x\right)^{2} \\
& \leq \frac{1}{2}(\Delta-\gamma) \frac{1}{\int_{a}^{b} w(y) d y} \int_{a}^{b} w(x)\left|f(x)-\frac{1}{\int_{a}^{b} w(y) d y} \int_{a}^{b} w(y) f(y) d y\right| d x \\
& \left(\leq \frac{1}{4}(\Gamma-\gamma)^{2}\right)
\end{aligned}
$$

provided $-\infty<\gamma \leq f(x) \leq \Gamma<\infty$ for a.e. $x \in[a, b]$. The constant $\frac{1}{2}$ is sharp.

If $w(x)=1, x \in[a, b]$, then we recapture the result in [4] as depicted here by (1.5).

2. Let $\overline{\mathbf{a}}=\left(a_{1}, \ldots, a_{n}\right), \overline{\mathbf{b}}=\left(b_{1}, \ldots, b_{n}\right), \overline{\mathbf{p}}=\left(p_{1}, \ldots, p_{n}\right)$ be $n$-tuples of real numbers with $p_{i} \geq 0(i \in\{1, \ldots, n\})$ and $\sum_{i=1}^{n} p_{i}=1$. If

$$
b \leq b_{i} \leq B, \quad i \in\{1, \ldots, n\},
$$

then one has the inequality

$$
\begin{aligned}
\left|\sum_{i=1}^{n} p_{i} a_{i} b_{i}-\sum_{i=1}^{n} p_{i} a_{i} \cdot \sum_{i=1}^{n} p_{i} b_{i}\right| & \leq \frac{1}{2}(B-b) \sum_{i=1}^{n} p_{i}\left|a_{i}-\sum_{j=1}^{n} p_{j} a_{j}\right| \\
& \leq \frac{1}{2}(B-b)\left[\sum_{i=1}^{n} p_{i}\left|a_{i}-\sum_{j=1}^{n} p_{j} a_{j}\right|^{p}\right]^{\frac{1}{p}} \text { if } 1<p<\infty \\
& \leq \frac{1}{2}(B-b) \max _{i=1, n}\left|a_{i}-\sum_{j=1}^{n} p_{j} a_{j}\right|
\end{aligned}
$$

The constant $\frac{1}{2}$ is sharp in the first inequality.

If $p_{i}=1, i \in\{1, \ldots n\}$, the following unweighted inequality may be stated

$$
0 \leq \frac{1}{n} \sum_{i=1}^{n} a_{i} b_{i}-\frac{1}{n} \sum_{i=1}^{n} a_{i} \cdot \frac{1}{n} \sum_{i=1}^{n} b_{i}
$$




$$
\begin{aligned}
& \leq \frac{1}{2}(B-b) \frac{1}{n} \sum_{i=1}^{n}\left|a_{i}-\frac{1}{n} \sum_{j=1}^{n} a_{j}\right| \\
& \leq \frac{1}{2}(B-b)\left(\frac{1}{n} \sum_{i=1}^{n}\left|a_{i}-\frac{1}{n} \sum_{j=1}^{n} a_{j}\right|^{p}\right)^{\frac{1}{p}} \\
& \leq \frac{1}{2}(B-b) \max _{i=\overline{1, n}}\left|a_{i}-\frac{1}{n} \sum_{j=1}^{n} a_{j}\right|
\end{aligned}
$$

The following counterpart of Schwarz's inequality also holds

$$
\begin{aligned}
0 & \leq \sum_{i=1}^{n} p_{i} a_{i}^{2}-\left(\sum_{i=1}^{n} p_{i} a_{i}\right)^{2} \leq \frac{1}{2}(A-a) \sum_{i=1}^{n} p_{i}\left|a_{i}-\sum_{j=1}^{n} p_{j} a_{j}\right| \\
& \left(\leq \frac{1}{4}(A-a)^{2}\right)
\end{aligned}
$$

provided $a \leq a_{i} \leq A$ for each $i \in\{1, \ldots, n\}$ and $\sum_{i=1}^{n} p_{i}=1$. The constant $\frac{1}{2}$ is sharp.

\section{Applications for Ostrowski's inequality}

If $\varphi:[a, b] \rightarrow \mathbb{R}$ is an absolutely continuous function on $[a, b]$ such that $\varphi^{\prime} \in L_{\infty}[a, b]$, then the following inequality is known in the literature as Ostrowski's inequality

$$
\begin{aligned}
\mid \varphi(x) & -\frac{1}{b-a} \int_{a}^{b} \varphi(t) d t \mid \\
\leq & {\left[\frac{1}{4}+\left(\frac{x-\frac{a+b}{2}}{b-a}\right)^{2}\right]\left\|\varphi^{\prime}\right\|_{\infty}(b-a), \quad x \in[a, b], }
\end{aligned}
$$

where $\left\|\varphi^{\prime}\right\|_{\infty}:=e s s \sup _{\alpha \in[a, b]}\left|\varphi^{\prime}(x)\right|$. The constant $\frac{1}{4}$ is best possible.

A simple proof of this fact, as mentioned in [1], may be accomplished by the use of the Montgomery identity

$$
\varphi(x)=\frac{1}{b-a} \int_{a}^{b} \varphi(t) d t+\frac{1}{b-a} \int_{a}^{b} K(x, t) \varphi^{\prime}(t) d t,
$$

where the kernel $K:[a, b]^{2} \rightarrow \mathbb{R}$ is defined by

$$
K(x, t):= \begin{cases}t-a \text { if } & a \leq t \leq x \\ t-b \text { if } & a \leq x<t \leq b\end{cases}
$$


We will now use the unweighted version of the inequality (3.2), namely, (1.5) (obtained by Cheng and Sun [4]) to procure the next result concerning a perturbed version of Ostrowski's inequality (4.1).

The following result also obtained by Cheng $[3]$ is recaptured in a simpler manner. A weighted version of this result was obtained by Roumeliotis [5].

Theorem 3. Assume that $\varphi:[a, b] \rightarrow \mathbb{R}$ is an absolutely continuous function on $[a, b]$ such that $\varphi^{\prime}:[a, b] \rightarrow \mathbb{R}$ satisfies the condition

$$
-\infty<\gamma \leq \varphi^{\prime}(x) \leq \Gamma<\infty \text { for a.e. } x \in[a, b] .
$$

Then we have the inequality

$$
\left|\varphi(x)-\frac{1}{b-a} \int_{a}^{b} \varphi(t) d t-\left(x-\frac{a+b}{2}\right)[\varphi ; a, b]\right| \leq \frac{1}{8}(b-a)(\Gamma-\gamma)
$$

for any $x \in[a, b]$, where $[\varphi ; a, b]=\frac{\varphi(b)-\varphi(a)}{b-a}$ is the divided difference. The constant $\frac{1}{8}$ is best possible.

Proof. We apply inequality (3.1) for the choices $w(t)=1, f(t)=K(x, t)$ defined by (4.3), $g(t)=\varphi^{\prime}(t), t \in[a, b]$ to get

$$
\begin{gathered}
\left|\frac{1}{b-a} \int_{a}^{b} K(x, t) \varphi^{\prime}(t) d t-\frac{1}{b-a} \int_{a}^{b} K(x, t) d t \cdot \frac{1}{b-a} \int_{a}^{b} \varphi^{\prime}(t) d t\right| \\
\quad \leq \frac{1}{2}(\Gamma-\gamma) \cdot \frac{1}{b-a} \int_{a}^{b}\left|K(x, t)-\frac{1}{b-a} \int_{a}^{b} K(x, s) d s\right| d t .
\end{gathered}
$$

We obviously have,

$$
\frac{1}{b-a} \int_{a}^{b} K(x, t) d t=x-\frac{a+b}{2}
$$

and

$$
\frac{1}{b-a} \int_{a}^{b} \varphi^{\prime}(t) d t=\frac{\varphi(b)-\varphi(a)}{b-a}
$$

Also

$$
\begin{aligned}
I(x) & :=\frac{1}{b-a} \int_{a}^{b}\left|K(x, t)-\left(x-\frac{a+b}{2}\right)\right| d t \\
& =\frac{1}{b-a}\left[\int_{a}^{x}\left|t-a-x+\frac{a+b}{2}\right| d t+\int_{x}^{b}\left|t-b-x+\frac{a+b}{2}\right| d t\right] \\
& =\frac{1}{b-a}\left[\int_{a}^{x}\left|t-x+\frac{b-a}{2}\right| d t+\int_{x}^{b}\left|t-x-\frac{b-a}{2}\right| d t\right] .
\end{aligned}
$$


Straight forward substitution of $u=t-x+\frac{b-a}{2}$ and $v=t-x-\frac{b-a}{2}$ gives

$$
\begin{aligned}
I(x) & =\frac{1}{b-a}\left[\int_{\frac{a+b}{2}-x}^{\frac{b-a}{2}}|u| d u+\int_{-\frac{b-a}{2}}^{\frac{a+b}{2}-x}|v| d v\right] \\
& =\frac{1}{b-a} \int_{-\frac{b-a}{2}}^{\frac{b-a}{2}}|u| d u=\frac{2}{b-a} \int_{0}^{\frac{b-a}{2}} u d u=\frac{b-a}{4} .
\end{aligned}
$$

Substitution of the above into (4.6) produces (4.5). The sharpness of the constant was proved in [3].

\section{Application for the Generalised trapezoid inequality}

If $\varphi:[a, b] \rightarrow \mathbb{R}$ is an absolutely continuous function on $[a, b]$ so that $\varphi^{\prime} \in L_{\infty}[a, b]$, then the following inequality is known as the generalised trapezoid inequality

$$
\begin{gathered}
\left|(x-a) \varphi(a)+(b-x) \varphi(b)-\int_{a}^{b} \varphi(t) d t\right| \\
\leq\left[\frac{1}{4}(b-a)^{2}+\left(x-\frac{a+b}{2}\right)^{2}\right]\left\|\varphi^{\prime}\right\|_{\infty}
\end{gathered}
$$

for any $x \in[a, b]$. The constant $\frac{1}{4}$ is best possible.

A simple proof of this fact is accomplished by using the identity [2]

$$
\int_{a}^{b} \varphi(t) d t=(x-a) \varphi(a)+(b-x) \varphi(b)+\int_{a}^{b}(x-t) \varphi^{\prime}(t) d t .
$$

Utilising the inequality (3.1) we may point out the following perturbed version of (5.1).

Theorem 4. Assume that $\varphi:[a, b] \rightarrow \mathbb{R}$ is an absolutely continuous function on $[a, b]$ so that $\varphi^{\prime}:[a, b] \rightarrow \mathbb{R}$ satisfies the condition (4.4). Then we have the inequality

$$
\begin{aligned}
& \left|\frac{1}{b-a} \int_{a}^{b} \varphi(t) d t-\left[\left(\frac{x-a}{b-a}\right) \varphi(a)+\left(\frac{b-x}{b-a}\right) \varphi(b)\right]-\left(x-\frac{a+b}{2}\right)[\varphi ; a, b]\right| \\
& \quad \leq \frac{1}{8}(b-a)(\Gamma-\gamma)
\end{aligned}
$$

for any $x \in[a, b]$, where $[\varphi ; a, b]$ is the divided difference. The constant $\frac{1}{8}$ is sharp.

Proof. We apply inequality (3.2) for the choices $f(t)=(x-t), g(t)=\varphi^{\prime}(t), w(t)=$ $1, t \in[a, b]$, to get

$$
\left|\frac{1}{b-a} \int_{a}^{b}(x-t) \varphi^{\prime}(t) d t-\frac{1}{b-a} \int_{a}^{b}(x-t) d t \cdot \frac{1}{b-a} \int_{a}^{b} \varphi^{\prime}(t) d t\right|
$$




$$
\leq \frac{1}{2}(\Gamma-\gamma) \frac{1}{b-a} \int_{a}^{b}\left|(x-t)-\frac{1}{b-a} \int_{a}^{b}(x-s) d s\right| d t
$$

Since

$$
\begin{aligned}
& \frac{1}{b-a} \int_{a}^{b}(x-t) d t=\left(x-\frac{a+b}{2}\right) \\
& \frac{1}{b-a} \int_{a}^{b} \varphi^{\prime}(t) d t=\frac{\varphi(b)-\varphi(a)}{b-a}=[\varphi ; a, b]
\end{aligned}
$$

and

$$
\begin{aligned}
\frac{1}{b-a} \int_{a}^{b}\left|(x-t)-\frac{1}{b-a} \int_{a}^{b}(x-s) d s\right| d t & =\frac{1}{b-a} \int_{a}^{b}\left|x-t-x+\frac{a+b}{2}\right| d t \\
& =\frac{1}{b-a} \int_{a}^{b}\left|t-\frac{a+b}{2}\right| d t \\
& =\frac{b-a}{4}
\end{aligned}
$$

from (5.4) we deduce the desired inequality (5.3).

The sharpness of the constant may be shown on choosing $x=\frac{a+b}{2}$ and $\varphi(t)=$ $\left|t-\frac{a+b}{2}\right|, t \in[a, b]$. We omit the details.

\section{References}

[1] P. Cerone and S.S. Dragomir, Midpoint-type rules from an inequalities point of view, Handbook of Analytic-Computational Methods in Applied Mathematics, Editor: G. Anastassiou, CRC Press, N.Y., (2000), 135-200.

[2] P. Cerone and S.S. Dragomir, Trapezoid-type rules from an inequalities point of view, Handbook of Analytic-Computational Methods in Applied Mathematics, Editor: G. Anastassiou, CRC Press, N.Y., (2000), 65-134.

[3] X. L. Cheng, Improvement of some Ostrowski-Grüss type inequalities, Computers Math. Applic., 42 (2001), 109-114.

[4] X. L. Cheng and J. Sun, A note on the perturbed trapezoid inequality, J. Ineq. Pure. Es Appl. Math., 3(2) (2002), Article 29. [ONLINE] http://jipam.vu.edu.au/v3n2/046_01.html

[5] J. Roumeliotis, Improved weighted Ostrowski-Grüss type inequalities, RGMIA Research Report Collection, 5(1) (2002), Article 13. [ONLINE] http://rgmia.vu.edu.au/v5n1.html

School of Computer Science and Mathematics, Victoria University, PO Box 14428, MCMC 8001, Victoria, Australia.

E-mail: pc@csm.vu.edu.au

School of Computer Science and Mathematics, Victoria University, MCMC 8001, Victoria, Australia.

E-mail: sever@csm.vu.edu.au 